\title{
PATTERN OF TEMPERATURE STRUCTURES IN HIMALAYAN SUBDUCTION ZONE: A REVIEW
}

\author{
Ismail Hossain* and Md. Rezaul Islam \\ Department of Geology and Mining, University of Rajshahi, Rajshahi 6205, Bangladesh \\ ${ }^{*}$ Corresponding author: Ismail Hossain \\ E-mail address: ismail_gm@ru.ac.bd (I. Hossain)
}

\begin{abstract}
The present study deals with the temperature structures (e.g., mantle wedge and slab) in Himalayan subduction zone. The estimated maximum temperature in the mantle wedge, $\operatorname{Tr}$ is $1420^{\circ} \mathrm{C}$, whereas temperature at the top of the slab, Ts ranges from $1081.3-1119.5^{\circ} \mathrm{C}$. The average value of the temperature and standard deviation at the top of the slab is $1103 \pm 10.7^{\circ} \mathrm{C}$. The study shows that the temperature of the mantle wedge is more or less stable and the slab temperature of the entire Himalayan belt is slightly varied. The mantle wedge temperature of the Himalayan subduction zone is correlated with Kamchatka subduction zone $\left(1450^{\circ} \mathrm{C}\right)$ and Tohoku subduction zone $\left(1400^{\circ} \mathrm{C}\right)$ in Northeast Japan. From overall observation, the Himalayan subduction zone is characterized with high compression and high seismic activity of the entire tectonic boundary along both the eastern and western sections. In these contexts, there might have a great possibility for large earthquakes to creep up in this region. The results of the research may contribute to explain geometry, rheology, heat transport and petrological processes of Himalayan subduction zone. Generally a temperature dependent process in the mantle wedge is responsible for the focusing of volcanic activity at the sharp fronts to the arcs.
\end{abstract}

Key words: Temperature structures, Himalayan subduction zone, volcanic activity, mantle wedge and slab, Himalaya-Tibetan orogen.

সারাংশঃ বর্তমান গবেষণায় হিমালয়ান সাবডাকশন জোনের তাপমাত্রা-বিন্যাস সম্পর্কে আলোকপাত করা হয়েছে। এতে পরিমাপকৃত ম্যান্টল ওয়েজের $\left(\mathrm{T}_{\mathrm{r}}\right)$ সর্বোচ্চ তাপমাত্রা

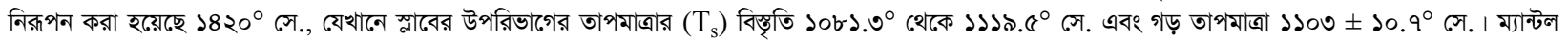
ওয়েজের তাপমাত্রা মোটামুটি স্থির হলেও স্লাবের উপরিভাগের তাপমাত্রা হিমালয়ের বিভিন্ন স্থানে কিছুটা ভিন্নতা দেখা যায়। এ ম্যান্টল ওয়েজের তাপমাত্রার সাথে কামচাট্কা সাবডাকশন জোন (১৪৫০০ সে.) এবং উত্তর-পূর্ব জাপানের তোহোকো সাবডাকশন জোনের (১৪০০ঃ সে.) কিছুটা মিল পরিলক্ষিত হয়। হিমালয়ান সাবডাকশন জোনের পূর্ব ও পশ্চিমের সামগ্রিক বিশ্লেষণে দেখা যায় এর উচ্চ চাপ (compression) এবং উচ্চ সিজমিক (seismic) ক্রিয়াশীলতা টেকটোনিক রেখা বরাবর প্রযুক্ত রয়েছে। এসব লক্ষণ থেকে ধারণা করা যায় এ অঞ্চলে যে কোন সময়ে বড় ধরনের ভূমিকম্প হওয়ার আশঙ্কা রয়েছে। হিমালয়ান সাবডাকশন জোনের উপর প্রস্তাবিত গবেষণার ফলাফল জ্যামিতিক বিশ্লেষণ, এর গতিশীলতার বৈশিষ্ট্য, তাপমাত্রা স্থানান্তর এবং শিলাতাত্ত্বিক প্রক্রিয়ার বিশ্লেষণে ভূমিকা রাখতে পারে। এছাড়া ম্যান্টল ওয়েজের তাপমাত্রা-বিন্যাস পরবর্তীতে হিমালয় পর্বত শ্রেণীতে আগ্নেয়গিরির জ্বালামুখ গঠন প্রক্রিয়ায় ভূমিকা পালন করতে পারে।

\section{Introduction}

The Himalayan subduction zone is where Indian plate dives under Eurasian plate and the collision causes earthquakes along it and melts the rocks (Fig. 1). Several major earthquakes have occurred at the plate interiors and boundaries in the Indian subcontinent during the last three decades (Sridevi 2004). The molten rock may also have enough pressure to break the surface to form magma chambers or volcanoes. Recently England et al. (2004) observed that the depth of the top of the intermediate depth seismicity beneath arc volcanoes (lying close to the top of the slab) varies systematically with the speed of decent of the slab has motivated them to seek the scaling relations for temperature structure in subduction zone in order to develop understanding of this systematic variation, and to provide a simple framework in which to investigate its implication for the process of melting and melt transport in subduction zone. So, how much temperature of the top of slab and the mantle wedge along the Himalayan range are varied with convergence rates, depth to the mantle wedge and the top of the slab, and the angle of the subducting slab, and associated plate deformation? This general inquiry is the leading motivator to instigate the study. As one of the largest mountain belts, the modern Himalaya-Tibetan orogen is a natural laboratory for investigating the distribution of melt in an active orogen and for studying the melt products during its growth (Jamieson et al. 2011).

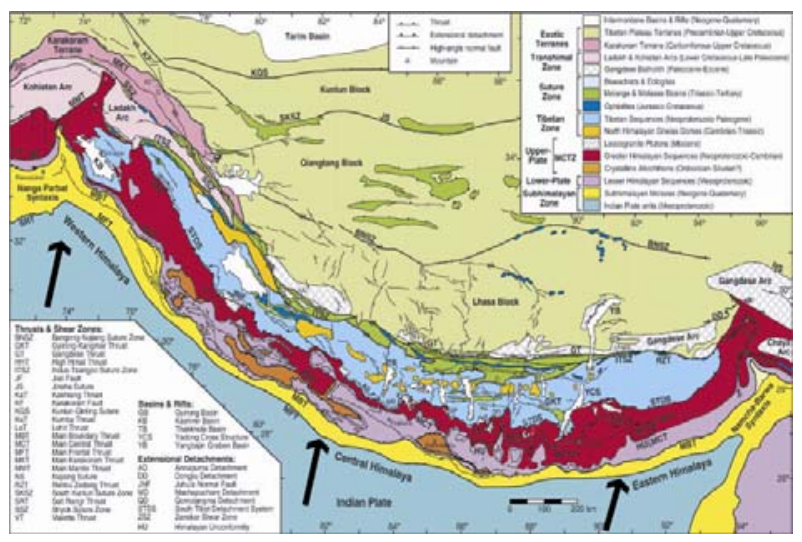

Figure1 Location of the entire area of the Himalayan subduction zone with simplified geology of the Himalayan Orogen, showing main tectonostratigraphic rock units and major structures (slightly modified from Goscombe et al. 2006). 
Similar observation from other active mountain belts has been accomplished at different subduction zones in the world like the Aleutian-Alaska subduction zone (Abers et al. 2005), Costa Rica-Nicaragua subduction zone (Peacock et al. 2004), Kamchatka subduction zone (Portnyagin and Manea 2008), Cocos plate under the Caribbean plate at the Nicoya Peninsula, Costa Rica (Harris and Wang 2002), Central Andean subduction zone (Springer and Föster 1998) but in our study area this type of approximation is not available, except some of related studies (e.g., Bollinger et al. 2004; Bilham et al. 1997a; Bilham 2008; Sridevi 2004; Catherine 2004). So, these observations facilitate to provide sufficient interest to review the temperature structures in Himalayan subduction zone.

\section{Methods}

There are various data set based on the variable convergence rates in place to place in the Himalayan subduction zone, and those data were collected from various published sources (Sridevi 2004; Bilham et al. 1997a; Bilham et al. 1997b; Bilham et al. 2001; Bollinger et al. 2006; Paul et al. 2001; Parkash et al. 2011; Johnson 2001; Peltzer and Saucier 1996; Wesnovsky et al. 1999; Molnar 1987) for reviewing in detail temperature structures of the Himalayan subduction zone. To evaluate the temperature structures along the Himalayan subduction zone, it is necessary to resolve the maximum temperature in the mantle wedge, $\mathrm{Tr}$ and the temperature at the top of the slab, Ts. The estimation of these two parameters ( $\mathrm{Tr}$ and Ts) is done using a simple mathematical model from England and Katz (2010). Although calculations of the full temperature field in subduction zones require numerical models, their results can be encapsulated in simple scaling relations that show that temperatures within the mantle wedge and at the top of the slab depend upon a single parameter, $\mathrm{Vr} \delta^{2} / \mathrm{k}$ (Fig. 2a, 2b). The maximum temperature in the mantle wedge, $\operatorname{Tr}$ is given by-

$$
\operatorname{Tr}=\mathrm{To} \exp \left(-\mathrm{B}\left(\mathrm{Vr} \delta^{2} / \mathrm{k}\right)^{\beta}\right) \ldots \ldots \ldots(1) \text { and the }
$$

temperature at the top of the slab, Ts is-

$$
\mathrm{Ts}=\mathrm{Tr} / 1+\mathrm{C}\left(\mathrm{Vr}^{2} / \mathrm{k}\right)^{\gamma} \ldots \ldots .(2)
$$

The required dimensionless parameter, $\mathrm{vr}^{2} / \mathrm{k}$ for evaluating the maximum temperature in the mantle wedge can be written as-

$$
\mathrm{Vr} \delta^{2} / \mathrm{k}=\mathrm{VR}_{\mathrm{D}} \delta^{2} / \mathrm{k}=\mathrm{V} \delta^{2}\left(\mathrm{D}-\mathrm{Z}_{\mathrm{W}}\right) / \mathrm{k} \operatorname{Sin}(\delta)
$$

Then, the radial distance from the wedge corner-

$$
\mathrm{r}=\mathrm{R}_{\mathrm{D}}=\mathrm{D}-\mathrm{Z}_{\mathrm{W}} / \operatorname{Sin}(\delta)
$$

Table 1 Notation with expressions for, or values of, parameters.

\begin{tabular}{|l|l|c|}
\hline Symbol & \multicolumn{1}{|c|}{ Definition } & \multicolumn{1}{c|}{ Notation/value } \\
\hline $\mathrm{k}$ & Thermal diffusivity & $\begin{array}{c}8 \times 10^{-7} \mathrm{~m}^{2} / \mathrm{s} \\
(\text { England and Katz 2010) }\end{array}$ \\
\hline $\mathrm{V}$ & Convergence rate & Variable \\
\hline$\delta$ & $\begin{array}{l}\text { Radial distance from } \\
\text { wedge corner }\end{array}$ & $?$ \\
\hline To & Dip of the slab & $\begin{array}{c}14-19^{\circ} \text { and } 20^{\circ} \\
(\text { Kaneko et al. 2003; } \\
\text { Jamieson et al. 2011). }\end{array}$ \\
\hline $\mathrm{B}, \mathrm{C}$ & Constants & $\begin{array}{c}1420^{\circ} \mathrm{C} \\
(\text { England and Katz 2010) }\end{array}$ \\
\hline$\beta, \gamma$ & $\begin{array}{c}\text { Constants } \\
(\text { England and Katz 2010) }\end{array}$ \\
\hline Tr & $\begin{array}{l}\text { Maximum } \\
\text { temperature in } \\
\text { the mantle wedge }\end{array}$ & $\begin{array}{c}-0.8,-0.06 \\
(\text { England and Katz 2010) }\end{array}$ \\
\hline Ts & $\begin{array}{l}\text { Temperature at } \\
\text { the top of the slab }\end{array}$ & $?$ \\
\hline
\end{tabular}

Using the parameters mentioned in Table 1, we estimated the maximum temperature in the mantle wedge, $\mathrm{Tr}$ and the temperature at the top of the slab, Ts with the help of the equations 1 and 2, and a program have been prepared using MATLAB (Version, 7.8.0.347, R2009a) to calculate the maximum mantle wedge temperature $\mathrm{Tr}$ and the slab temperature at the top of the slab, Ts, and the results are shown in Table 2 and Table 3.

\section{Regional Tectonics}

The Indian craton was once part of the supercontinent of Pangea. At that time, it was attached to Madagascar and Southern Africa on the south west coast, and Australia along the east coast 160 Ma (ICS 2004) during the Jurassic period, rifting caused Pangea to break apart into supercontinents namely, Gondwana (to the south) and Laurasia (to the north). The Indian craton remained attached to Gondwana, until the supercontinent began to break apart in the Early Cretaceous, about 125 Ma (ICS 2004). The Indian plate then drifted northward toward the Eurasian plate, at a place that is the fastest movement of any known plate. It is generally believed that the Indian plate separated from Madagascar about $90 \mathrm{Ma}$; however some biogeographical and geological evidences suggest that the connection between Madagascar and Africa was retained at the time when the Indian plate collided with the Eurasian plate about $50 \mathrm{Ma}$ (ICS 2004). General consensus that the crustal thickening driven by collision between India and Asia began 50-55 Ma ago. The Indian plate and its leading edge now lies under the 
Tibetan plateau, about $300 \mathrm{~km}$ north of the IndusTsangpo suture zone that marks the surface expression of the collision boundary (Nábělek et al. 2009). Plate reconstructions show that the convergence velocity between Indian subcontinent and Eurasia changed dramatically 50-35 Ma (Copley et al. 2010). The rate of convergence started decreasing at about the time of the initial contact of the Indian and Asian continental margins and the cessation of marine sedimentation in the western Himalayas (Guillot et al. 2003). The relative velocity continued to decrease as the Himalayan orogen developed and the Tibetan Plateau was uplifted. Rowley and Currie (2006) suggest the Tibetan Plateau had attained close to its current elevation at $35 \mathrm{Ma}$, after which the Indian plate motion stabilized. This orogeny, which is continuing today, is related to closure of the Tethys Ocean. The closure of this ocean which created the Alps in Europe, and the Caucasus range in western Asia, created Himalaya Mountains and the Tibetan Plateau in South Asia.
In the Early Tertiary, the first phase of the Himalayan Orogeny, the Karakoran phase occurred, the Himalayan orogeny is continuing to the present day (ICS 2004). The current orogenic event is causing of the Asian to deform westward and eastward on either side of the orogeny. The present day crustal thickness beneath the Himalaya exceeds $80 \mathrm{~km}$, and inferred temperatures in the middle to lower crust are well above $700^{\circ} \mathrm{C}$ (Nelson et al. 1996; Klemperer 2006). Geological and geophysical evidences indicate that melting began at about $30 \mathrm{Ma}$ and continues till this day (Jamieson et al. 2011).

\section{Results}

Analyses have been carried out considering the convergence rates, depth to the mantle wedge and the top of the slab, and the angle of the subducting slab along the Himalayan subduction zone for getting temperature structures (Table 2 and Table 3 ).

Table 2 Estimated maximum temperature in the mantle wedge and the temperatures at the top of the slab depending on the maximum and minimum value of $\mathrm{D} \approx 80-160 \mathrm{~km}, \mathrm{Zw} \approx 40-80 \mathrm{~km}$, and $\delta \approx 14-19^{\circ}$.

\begin{tabular}{|c|c|c|c|c|c|}
\hline $\begin{array}{c}\text { Convergenc } \\
\text { e rate (V) } \\
\mathrm{mm} / \mathrm{yr}\end{array}$ & Region & Source & $\begin{array}{c}\text { Max. } \\
\text { temperature in } \\
\text { the mantle } \\
\text { wedge, } \operatorname{Tr}^{\circ} \mathrm{C} \\
\end{array}$ & $\begin{array}{l}\text { Max. temperature at the } \\
\text { top of the slab, Ts }{ }^{\circ} \mathrm{C}\end{array}$ & $\begin{array}{c}\text { Min. } \\
\text { temperature at } \\
\text { the top of the } \\
\text { slab, Ts }{ }^{\circ} \mathrm{C} \\
\end{array}$ \\
\hline $10-20$ & $\begin{array}{l}\text { Kashmir to } \\
\text { Arunachal }\end{array}$ & Sridevi, 2004 & 1420 & 1116.32 & 1086.74 \\
\hline $10-12$ & Sikkim & , & 1420 & 1108.94 & 1086.74 \\
\hline $10-18$ & Garhwal & 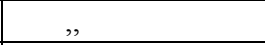 & 1420 & 1114.81 & 1086.74 \\
\hline $10-18$ & Kumaun & , & 1420 & 1114.81 & 1086.74 \\
\hline $14-20$ & Ladakh & , & 1420 & 1116.32 & 1091.86 \\
\hline $20 \pm 5$ & Lhasa & " & 1420 & 1119.51 & 1092.91 \\
\hline $20 \pm 3$ & \multirow[t]{2}{*}{ Tibet } & Bilham et al. 1997a & 1420 & 1118.32 & 1094.79 \\
\hline $18.5 \pm 2$ & & Bilham et al. 1997b & 1420 & 1116.47 & 1094.07 \\
\hline $16-20$ & & Bilham et al. 2001 & 1420 & 1116.32 & 1093.88 \\
\hline $21 \pm 1.5$ & \multirow{2}{*}{ Along MFT } & Bollinger et al. 2006 & 1420 & 1118.01 & 1096.85 \\
\hline $18 \pm 7$ & & Paul et al. 2001 & 1420 & 1118.76 & 1088.19 \\
\hline $21.5 \pm 1.5$ & \multirow[t]{2}{*}{ Hardwar } & Parkash et al. 2011 & 1420 & 1118.32 & 1097.23 \\
\hline $15-20$ & & Johnson 2001 & 1420 & 1116.32 & 1092.9 \\
\hline 13 & Pakistan & Johnson 2001 & 1420 & 1110.11 & 1090.73 \\
\hline $9-14$ & $\begin{array}{c}\text { Central Plateau, } \\
\text { Pakistan } \\
\end{array}$ & $"$ & 1420 & 1111.19 & 1085.13 \\
\hline 7 & $\begin{array}{c}\text { Eastern Plateau, } \\
\text { Pakistan }\end{array}$ & " & 1420 & 1101.02 & 1081.25 \\
\hline 18 & Nepal, Assam & $\begin{array}{l}\text { Peltzer and Saucier } \\
1996\end{array}$ & 1420 & 1114.81 & 1095.65 \\
\hline 10 & Jammu & , & 1420 & 1106.28 & 1086.74 \\
\hline 17.2 & Nepal & Bilham et al. 1997b & 1420 & 1114.16 & 1094.97 \\
\hline $13.8 \pm 3.6$ & Central India & Wesnovsky et al. 1999 & 1420 & 1114.33 & 1087.04 \\
\hline $18 \pm 7$ & Central India & Molnar 1987 & 1420 & 1119.51 & 1088.2 \\
\hline
\end{tabular}


Table 3. Estimated maximum temperature in the mantle wedge and the average temperature at the top of the slab depending on the average value of $\mathrm{D} \approx 120 \mathrm{~km}, \mathrm{Zw} \approx 60 \mathrm{~km}, \delta \approx 16.5^{\circ}$.

\begin{tabular}{|c|c|c|c|}
\hline Convergence rate, $\mathbf{V ~ m m} / \mathbf{y r}$ (Average) & Region & $\begin{array}{c}\text { Maximum temperature in the } \\
\text { mantle wedge, } \mathbf{T r} \mathbf{~}^{\mathbf{C}}\end{array}$ & $\begin{array}{c}\text { Average temperature at the } \\
\text { top of the slab, } \mathbf{T s} \mathbf{}^{\mathbf{}} \mathbf{C}\end{array}$ \\
\hline 15 & Kashmir to Arunachal & 1420 & 1103.86 \\
\hline 11 & Sikkim & 1420 & 1099.27 \\
\hline 14 & Garhwal & 1420 & 1102.84 \\
\hline 14 & Kumaun & 1420 & 1102.84 \\
\hline 17 & Ladakh & 1420 & 1105.71 \\
\hline 20 & Lhasa & 1108.08 \\
\hline 20 & Tibet & 1420 & 1108.08 \\
\hline 18.5 & & 1420 & 1106.94 \\
\hline 18 & & 1420 & 1106.54 \\
\hline 21 & Along MFT & 1420 & 1108.80 \\
\hline 18 & & 1420 & 1106.54 \\
\hline 21.5 & Hardwar & 1420 & 1109.14 \\
\hline 17.5 & & 1420 & 1106.13 \\
\hline 13 & Pakistan & 1420 & 1101.75 \\
\hline 11.5 & Central Plateau, Pakistan & 1420 & 1099.93 \\
\hline 7 & Eastern Plateau, Pakistan & 1420 & 1092.48 \\
\hline 18 & Nepal, Assam & 1420 & 1106.54 \\
\hline 10 & Jammu & 1420 & 1097.84 \\
\hline 17.2 & Nepal & 1105.88 \\
\hline 13.8 & Central India & 1420 & 1102.63 \\
\hline 18 & Central India & 1420 & 1106.54 \\
\hline
\end{tabular}

The convergence rate in Ladakh ranges from 14-20 $\mathrm{mm} / \mathrm{yr}$ and the Ts ranges from $1092-1116^{\circ} \mathrm{C}$ whereas in Lhasa it is $20 \pm 3 \mathrm{~mm} / \mathrm{yr}$ and the Ts value range from 1093$1120^{\circ} \mathrm{C}$ (Sridevi 2004). The rate of slip of India beneath Tibet is found within the range of $20.5 \pm 2 \mathrm{~mm} / \mathrm{yr}$ and the related Ts value ranges from $1095-1118^{\circ} \mathrm{C}$ (Bilham et al. 1997b). Similarly the convergence rate in Sikkim is 10-20 $\mathrm{mm} / \mathrm{yr}$ and Ts ranges from $1087-1109^{\circ} \mathrm{C}$, in the case of Garhwal and Kumaun the convergence rate ranges from $10-18 \mathrm{~mm} / \mathrm{yr}$ and their Ts ranges from $1087-1115^{\circ} \mathrm{C}$. Along the main frontal thrust the convergence rate is $21 \pm 1.5 \mathrm{~mm} / \mathrm{yr}$ and the Ts shows $1097-1118^{\circ} \mathrm{C}$ (Bollinger et al. 2006). Overall convergence of 10-20 mm/yr occurs in the $2500 \mathrm{~km}$ stretch of the Himalayan arc from Kashmir to Arunachal and the convergence rates vary from west to east and their related top of the slab temperature Ts vary from $1087-1116^{\circ} \mathrm{C}$. In general, the rate of convergence between Indian subcontinent and Eurasia over the period since initial collision was roughly $50 \mathrm{~mm} / \mathrm{yr}$. However, the possible long-term rate of convergence within the Himalaya is considerably less, i.e., $15-20 \mathrm{~mm} / \mathrm{yr}$, a fraction of the total convergence (Johnson et al. 2001). Avouac et al. (1998) and Wesnousky et al. (1999) quoted rate of $21 \pm 1$ and $13 \pm 3.6 \mathrm{~mm} / \mathrm{yr}$ respectively, for slip on the active Himalayan Main Frontal Thrust.

An approximation to the $50 \mathrm{~mm} / \mathrm{yr}$ plate convergence rate is achieved by adding shortening rates in regions north of the Himalaya (Tibet, Tien Shan, Kun Kun etc., Dewey et al. 1988). The problem with this is that it assumes that distributed shortening by thrust $/ Z$ fold tectonics has operated since the time of collision. But we know that is not true. For example, the Tien Shan is a Neogene (post 24 Ma) orogen (Hendrix et al. 1994). The inferences are either that the shortening rate in the Himalaya was much higher before the Miocene or that strike slip faulting operated over wide area of South East Asia.

After plotting convergence rate of corresponding region along the Himalayan subduction zone it is shown that the slab temperatures are slightly varied (Fig. 3). The maximum slab temperature is observed in Lhasa which is far away from the MBT and the minimum slab temperature is observed at Eastern plateau in Pakistan.

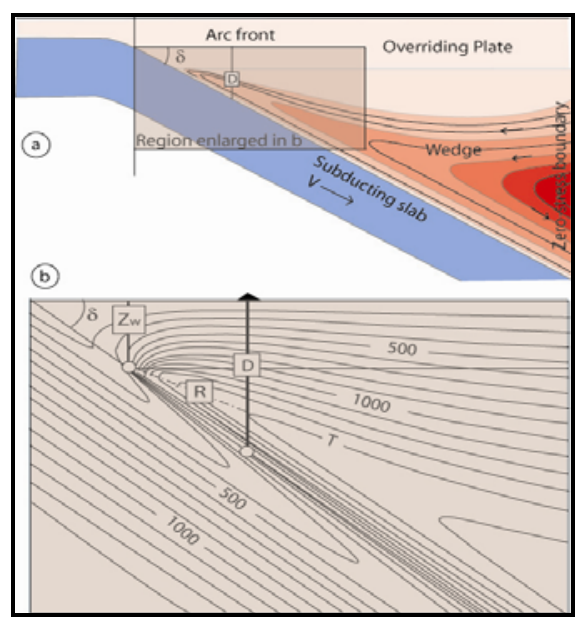

Figure 2. (a) Idealized cross-sections of a subduction zone. Two plates converge at a speed $\mathrm{V}$, with the slab of Indian plate (lithosphere) being subducted at an angle $\delta$ beneath the overriding plate. (b) Enlargement of temperature structure of the rectangle in a: Isotherms are shown at intervals of $100^{\circ} \mathrm{C}$. A schematic isotherm, labeled $\mathrm{T}$, has its closest approach to the wedge corner (its 'nose') immediately beneath the volcanic front, at a distance $\mathrm{R}$ from the corner (black circle). 


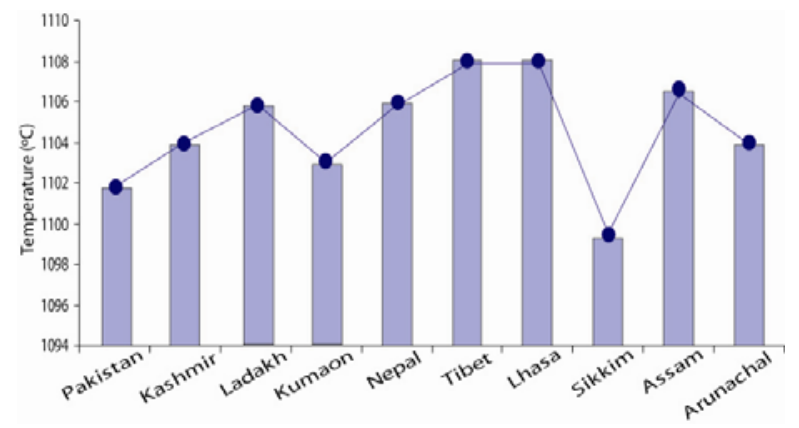

Figure 3 Maximum slab temperature distributions at the top of the slab along the Himalayan Range (in Line and in Histogram).

After plotting maximum slab temperature of corresponding places along the Himalayan subduction zone it is shown that the slab temperature is comparatively higher in central region (except Sikkim) than the Eastern and Western sides of the Himalayan region (Fig. 3). Comparatively low Ts value in Sikkim area may be the reflection of De and Kayal (2004) observation. They observed no remarkable seismic activity along NNW-SSE trending large Gangtok lineament (which indicate locally this area seismically less active).

\section{Discussions and Conclusions}

The results of the arc front temperature and geological data demonstrate a profound effect on the rock strength, that melt-weakening strongly influences the tectonic style of mountain belts that resultant weak partial-melt zones and magma arc present within large modern orogens. Both modern and ancient orogens belts display the protected interaction among melting deformation and crustal flow.

The analysis of the results gives the value of temperature structures (mantle wedge and slab) in Himalayan subduction zone, where maximum temperature in the mantle wedge, $\operatorname{Tr}$ is $1420^{\circ} \mathrm{C}$. This result in the study area doesn't depend upon the changes of convergence rates, depth to the mantle wedge and the top of the slab, and the angle of the subducting slab, whereas temperature at the top of the slab in the study area, Ts, ranges from 1081.3$1119.5^{\circ} \mathrm{C}$, which depend upon the changes of convergence rates, depth to the mantle wedge and the top of the slab, and the angle of the subducting slab. The mean value of the slab temperature is about $1103.3^{\circ} \mathrm{C}$ (Standard deviation is $\pm 10.7^{\circ} \mathrm{C}$ ).

According to Kelemen et al. (2003), thermal structure in the mantle wedge beneath arcs is almost $1450^{\circ} \mathrm{C}$. So, the result suggests that there might have a great possibility to develop volcanic arc in the studied mantle wedge, where the temperature is $1420^{\circ} \mathrm{C}$. The maximum temperature in the mantle wedge which is achieved in the study is consistent with Tohoku subduction zone, $1400^{\circ} \mathrm{C}$ in Northeast Japan (Honda 1985), Kamchatka subduction zone, $1450^{\circ} \mathrm{C}$ in Mexico (Manea et al. 2004).

From the analysis it is shown that the mantle wedge temperature along the Himalayan range is almost uniform whereas result of Ts shows slightly variable pattern (Grujic et al. 2006). It is remarkable that the slab temperatures variations are not so much high. The maximum slab temperature is $1119.51^{\circ} \mathrm{C}$ which is found in Lhasa, a great distance apart from MBT and the minimum slab temperature is $1081.25^{\circ} \mathrm{C}$ which is found at Eastern plateau in Pakistan. Generally plate convergence at rates of $10-50 \mathrm{~mm} / \mathrm{yr}$ initially forms small cold orogens with back to back wedges consisting largely of low-grade sedimentary and volcanic rocks (e.g., Willett et al. 1993). As these orogens grow along the orogenic main sequence thickened crust heats up by radioactive decay on timescales of ca $20 \mathrm{Ma}$ (Jamieson et al. 2011).

From overall observation, the study shows that the slab temperature in Central Himalaya (except Sikkim) is comparatively higher than Eastern and Western Himalaya. High compression and seismic activity characterize the entire tectonic boundary along both the eastern and western sections of the thrust. As a result there might be great possibility to form volcanic front and generate earthquake. For example, active collision along the northwestern boundary at the foothills of the Himalayan Mountains have already caused the destructive October 8, 2005 earthquake of magnitude $7.6(\mathrm{Mw})$ in Pakistan (Pararas-Carayannis 2005) and June 15, 1897 earthquake of magnitude $8.7(\mathrm{Mw})$ in Assam, India (Mahapatra and Mohanty 2009). Again, according to Bilham et al. (2001) the convergence has been responsible for many large earthquakes along the Himalayan arc in the past and could likely to creep up large earthquakes in the future.

So, this result may help to explain the complex details of geometry, rheology, heat transport and petrological processes of Himalayan subduction zone. These data are also helpful about the processes governing the location of volcanic arcs which is controlled by a strongly temperature dependent process taking place either at the top of the slab or in the wedge of mantle beneath the arcs (England and Wilkins 2004). Generally a temperature dependent process in the mantle wedge is responsible for the focusing of volcanic activity at the sharp fronts to the arcs. So, this study may also provide strong constraints in petrological modeling of the generation of arc margins. 
Acknowledgement: We acknowledge the constructive reviews and comments of anonymous reviewer. Special thanks are due to Md. Liton Arefin for his software support.

\section{References}

Abers GA, van Keken PE, Kneller EA, Ferris A, Stachnik JC. 2005. The thermal structure of subduction zones constrained by seismic imaging: Implications for slab dehydration and wedge flow. Earth Planet Sci Let 241, 387-397.

Avouac JP, Lave J, Cattin R, Pandey MR, Tandukar RP. 1998. Active Mountain building in the Himalaya of Nepal.AGU 79, fall meeting suppl. F204.

Bilham R, Larson K, Freymueller J, Project Idyllhim members. 1997a. Indo-Asian Convergence Rates in the Nepal Himalaya. Nature 386, 61-66.

Bilham R, Larson K, Freymueller J, Members IP. 1997b. GPS measurements of present-day convergence across the Nepal Himalaya. Nature 386, 61-64.

Bilham R, Gaur VK, Molnar P. 2001. Himalayan Seismic Hazard. Science 293, 1441-1444.

Bilham R. 2008. Tsunamigenic middle earth. Nature Geosci 1, 211-212.

Bollinger L, Avouac JP, Beyssac O, Catlos EJ, Harrison TM, Grove M, Goffe B, Sapkota S. 2004. Thermal structure and exhumation history of the Lesser Himalaya in central Nepal. Tectonics 23, 1-19.

Bollinger L, Henry P, Avouac JP. 2006. Mountain building in the Nepal Himalaya: Thermal and kinematic model. Earth Planet Sci Let 244, 58-71.

Catherine JK. 2004. A preliminary assessment of internal deformation in the Indian Plate from GPS measurements. J Asian Earth Sci 23(4), 461-465.

Copley A, Avouac JP, Royer JY. 2010. India-Asia collision and the Cenozoic slowdown of the Indian plate: Implications for the forces driving plate motions. J Geophys Res 115, B03410, doi:10.1029/2009JB006634.

De R, Kayal JR. 2004. Seismic activity at the MCT in Sikkim Himalaya. Tectonophysics, 386, 243-284.

Dewey JF, Shackleton RM, Chang C, Sun Y. 1988. The tectonic evolution of the Tibetan plateau. Phil Trans Royal Soc, London A327, 379- 413 .

England PC, Katz RF. 2010. Melting above the anhydrous solidus controls the location of volcanic arcs. Nature 467, 700-702.

England PC, Wilkins C. 2004. A simple analytical approximation to the temperature structure in subduction zones. Geophys J Int 159, 1138-1154.

England PC, Engdahl ER, Thatcher W. 2004. Systematic variation in the depths of slabs beneath arc volcanoes. Geophys J Int 156, 377-379.

Goscombe B, Gray D, Hand M. 2006. Crustal architecture of the Himalayan metamorphic front in eastern Nepal. Gondwana Res 10, 232-255.

Guillot S, Garzanti E, Baratoux D, Marquer D, Mahéo G, de Sigoyer J. 2003. Reconstructing the total shortening history of the NW Himalaya, Geochem Geophys Geosyst 4(7), 1064, doi:10.1029/2002GC000484.

Grujic D, Coutand I, Bookhagen B, Bonnet S, Blythe A, Duncan C. 2006. Climatic forcing of erosion, landscape, and tectonics in the Bhutan Himalayas. Geol Soc Am 34, 801-804.

Harris NR, Wang K. 2002. Thermal models of the Middle America Trench at the Nicoya Peninsula, Costa Rica. Geophys Res Let 29, P X-(1-4).

Hendrix MS, Graham SA, Amory JY, Gombosurengiin B. 1996. Noyon Uul syncline, southern Mongolia: Lower Mesozoic sedimentary record of the tectonic amalgamation of central Asia. GSA Bull 108(10), 1256-1274.

Honda S. 1985. Thermal structure beneath Tohoku, Northeast Japan. Tectonophysics 112, 69-102.

International Commission on Stratigraphy (ICS). 2004. Geology of India. Access on 2011.07.02.
Jamieson RA, Unsworth MJ, Harris NBW, Rosenberg CL, Schulmann K. 2011. Crustal Melting and the Flow of Mountains. Elements 7(4), 253-260.

Johnson MRW. 2001. Shortening budgets and the role of continental subduction during the India-Asia collision. Earth-Sci Rev 59, 101-123.

Kaneko Y, Katayama I, Yamamoto H, Misawa K, Ishikawa M, Rehman HU, Kausar AB, Shiraishi K. 2003. Timing of Himalayan ultrahigh-pressure metamorphism: sinking rate and subduction angle of the Indian continental crust beneath Asia. J Meta Geo l 21, 589-599.

Kelemen PB, Rilling JI, Parmentier EM, Mehl L, Hacker BR. 2003. Thermal structure due to solid-state flow in the Mantle Wedge beneath Arcs. http://www.geol.ucsb.edu/faculty/hacker/viz/Kelemen03 Conve ction mantle wedge.pdf. Access on 2011.07.02

Klemperer SL. $200 \overline{6}$. Crustal flow in Tibet: geophysical evidence for the physical state of Tibetan lithosphere, and inferred patterns of active flow. In: Law RD, Searle MP, Godin L. (eds) Channel Flow, Ductile Extrusion and Exhumation of Lower Mid-crust in Continental Collision Zones. Geol Soc London Sp Pub 268,39-70.

Manea VC, Manea M, Kostoglodov V, Currie CA, Sewell G. 2004. Thermal structure, coupling and metamorphism in the Mexican subduction zone beneath Guerrero. Geophys J Int 158, 775-777.

Mahapatra AK, Mohanty DK. 2009. Earthquake Forecasting in Northeast India using Energy Blocked Model. American Geophysical Union, Fall Meeting 2009, abstract \#S51B-1429.

Molnar P. 1987. Inversion of profiles of uplift rates for the geometry of dip-slip faults at depth with examples from the Alps and the Himalaya. Annales Geophysics 5, 663-670.

Nelson KD, 27 coauthors. 1996. Partially molten middle crust beneath southern Tibet: Synthesis of project INDEPTH results. Science 274, 1684-1688.

Nábělek J, Hetényi G, Vergne J, Sapkota S, Kafle B, Jiang M, Su H, Chen J, Huang BS, Hi-CLIMB Team. 2009. Underplating in the Himalaya-Tibet collision zone revealed by the Hi-CLIMB experiment. Science 325, 1371-1374.

Pararas-Carayannis G. 2005. 'The Earthquake of 8 October 2005 in Northern Pakistan'. http://www.drgeorgepc.com/Earthquake2005Pakistan.html. Access on 2011.07.02.

Parkash B, Rathor RS, Pati P, Jakhmola RP, Singh S. 2011. Convergence rates along the Himalayan Frontal Thrust inferred from terraces at Chandidevi Temple Hill, Hardwar, Northwestern Himalaya. Current Sci 100, 707-712.

Paul J, Bürgmann R, Gaur VK, Bilham R, Larson KM, Ananda MB, Jade S, Mukal M, Anupama TS, Satyal G, Kumar D. 2001. The motion and active deformation of India. Geophys Res Let 28, 647-650.

Peacock SM, van Keken PE, Holloway SD, Hacker BR, Abers GA, Fergason RL. 2004. Thermal structure of the Costa RicaNicaragua subduction zone. Phys Earth Planet Inter 149, 187-200.

Peltzer G, Saucier F. 1996. Present-day kinematics of Asia derived from geologic fault rates. J Geophys Res 101, 27943-27956.

Portnyagin M, Manea VC. 2008. Mantle temperature control on composition of arc magmas along the Central Kamchatka Depression. Geology Geol Soc Am 36 (7), 519-522.

Rowley DB, Currie BS. 2006. Palaeo-altimetry of the late Eocene to Miocene Lunpola basin, central Tibet. Nature 439, 677-681.

Springer M, Förster A. 1998. Heat-flow density across the Central Andean subduction zone. Tectonophysics 291, 123-139.

Sridevi J. 2004. Estimates of plate velocity and crustal deformation in the Indian subcontinent using GPS geodesy. Current Sci 86(10), 1443-1448.

Wesnousky SG, Kumar S, Mohindra R, Thakur VC. 1999. Uplift and convergence along Himalayan Frontal Thrust of India. Tectonics 18, 967-976.

Willett S, Beaumont C, Fullsack P. 1993. Mechanical model for the tectonics of doubly vergent compressional orogens. Geology 21(4), 371-374.

Manuscript received on 7 May 2012 and revised on 7 December 2012 